\title{
Percepción de los docentes sobre el uso de las redes sociales en el aula de Primaria. Una experiencia en un centro educativo de la Región de Murcia.
}

\section{Teachers' perception relating to the use of social network in the primary classrooms. A real experience in a school located in Murcian Region.}

\author{
Pedro Juan Ibáñez Zapata; pedrojuan.ibanez@um.es \\ María del Mar Sánchez Vera; mmarsanchez@um.es
}

Universidad de Murcia

\section{Resumen}

El objetivo principal de esta investigación ha sido conocer la percepción que tienen los docentes sobre el uso de las redes sociales en un centro educativo de la Región de Murcia y si esta se modifica al implementar en el aula una experiencia con estas herramientas. Consta de tres etapas en las que se tratará de conocer la percepción inicial de los docentes, se diseñará una red social educativa para mostrar sus posibilidades y finalmente se analizará si esta experiencia ha influido en su pensamiento inicial. Aunque después de esta experiencia se aprecia un cambio significativo en la actitud respecto a la incorporación de las RRSS, queda patente que su implementación todavía queda alejada de las teorías que fundamentan sus beneficios dentro del contexto educativo.

Palabras clave: Web 2.0, redes sociales, educación primaria, docentes, actitud.
Abstract

The aim of this research, has been to realize the perception of a group of Murcian primary school teachers, relating to the use of social network and if their point of view changes once they have introduced this tool in any of their teaching activities. This research is based on three different stages, firstly, we try to figure out the teachers' initial perception, next we create an educational social network to show all its possibilities and finally, we analyze if this experience has influenced teachers' initial thinking.

Although this experience has proved a significant change in attitude regarding the incorporation of social networks, it is obvious that its implementation is far away from those theories which base their benefits into the educational system.

Keywords: Web 2.0, social networks, primary education, teachers, attitude. 


\section{INTRODUCCIÓN: LAS REDES SOCIALES COMO COMUNIDADES CREATIVAS}

Uno de los elementos favorecedores de la integración de las TIC en nuestra sociedad, viene determinado por la concepción de la Web 2.0 o Web Social. Desde que Darcy DiNucci creara el término y Tim O'Reilly la catalogara en 2005 como una "arquitectura de participación", donde por medio de una ética de colaboración entre los usuarios se consigue potenciar y favorecer el intercambio de información a través de la cooperación, convirtiendo al receptor en usuario activo y generador de nuevos contenidos (García, 2008), no se ha parado de generar nuevas herramientas capaces de aportar destacadas posibilidades dentro del ámbito educativo.

Pero si hay una aplicación de la Web 2.0 que ha ganado en popularidad entre los jóvenes en los últimos años son sin duda las redes sociales, ya que como señalan Espuny, González, Lleixá y Gisbert (2011, p.173), han supuesto "una auténtica revolución, tanto por su propia generalización como por la extensión de sus aplicaciones ulteriores", constituyéndose en "poderosos medios de expresión, comunicación e intercambio ágil de información multiformato entre usuarios, capaces de favorecer la socialización de los mismos, y de tejer los fundamentos de comunicaciones de aprendizaje creativas, orientadas a impulsar la inteligencia colectiva global" (Del Moral y Villalustre, 2012, p.41).

Sloep y Berlanga (2011) presentan dos tipos de redes sociales destinadas para un uso educativo; las redes de aprendizaje definidas como "entornos de aprendizaje en línea que ayudan a los participantes a desarrollar sus competencias colaborando y compartiendo información" (p.56) y otras plataformas como Facebook y Twitter, aunque no fueron creadas precisamente para este cometido.

El reto al que nos enfrentamos los educadores ante la variedad de redes sociales que existen, es el de favorecer los procesos educativos abiertos y tratar de dar respuesta a las inquietudes y necesidades que surgen a medida que estas herramientas van adentrándose en la cotidianidad de un alumnado, que viene haciendo uso de las mismas desde un punto de vista comunicativo y de relaciones sociales y no lo percibe como una posibilidad de aprendizaje personal. En este sentido Alemany (2009) señala que las redes sociales sí podrían ayudar a generar un aprendizaje efectivo porque proveen al estudiante de un entorno creativo, facilitan el contacto entre alumnos y profesores, rompen la barrera espaciotemporal y ofrecen a los estudiantes una conexión con el contexto del mundo real. Además, refuerza las relaciones entre los miembros de un grupo, desarrollando una visión compartida y el sentido de pertenencia y corresponsabilidad con el mismo, a la misma vez que logra una capacidad de independencia y responsabilidad individual, lo que "favorece procesos de generación de contenidos y de construcción del propio aprendizaje" (Camacho, 2010, p.95).

El simple hecho de que una red social ofrezca una serie de aplicaciones y servicios no garantiza una calidad óptima de la misma, pudiendo darse una red social pero no una red de aprendizaje. No obstante, desde el ámbito educativo, incorporar las redes sociales en el marco de estrategias metodológicas adecuadas nos permitirían generar actividades que favorezcan la adquisición de algunas de las competencias básicas como: la búsqueda y selección apropiada de los contenidos, su gestión y su transformación en información que 
compartiremos con el desarrollo del trabajo cooperativo y colaborativo a través de las llamadas comunidades de aprendizaje.

Otro de los aspectos fundamentales para la integración de estas tecnologías en el aula, depende de la competencia digital que posean los docentes, entendida por Prendes y Gutiérrez (2011, p.199) como aquella "que tiene que ver con la capacidad, el conocimiento y la actitud sobre el uso de tecnologías de la información y la comunicación en sus diversas funciones y contextos de aplicación", por lo que la formación del profesorado se convierte en fundamental para un desarrollo adecuado de la competencia digital del alumnado (Gutiérrez, Prendes y Castañeda, 2015), ya que "solo con profesores competentes digitalmente podremos formar alumnos competentes en este sentido" (p.248). Por otro lado, Marquès (2008) señala que una motivación y actitud positiva de los docentes hacia el uso de las TIC, crecerá conforme aumente su formación y su conocimiento sobre modelos eficaces que puedan utilizar con facilidad en su propio contexto y que además les ayude en su labor docente. A pesar de todo, Fernández (2012) señala que en la actualidad todavía existen docentes que presentan reticencias y miedos al uso de las redes sociales con fines educativos alegando: "pérdida de control en la clase, falta de preparación por parte de los profesores, miedo a no cubrir el contenido y falta de materiales preparados para usar en clase" (p.165). Además, existe cierta resistencia a los cambios que se producen en los modelos educativos y a la formación continua de los docentes implicados.

\section{LAS REDES SOCIALES EN EDUCACIÓN PRIMARIA}

Gutiérrez, Sánchez y Castañeda (2013, p.1), señalan el camino de porqué acercar estas herramientas al aula desde estas primeras etapas educativas, argumentando que el entorno tecnológico del alumnado de Educación Infantil y Primaria se ha modificado totalmente, y marcará significativamente su desarrollo personal, social y profesional. Por tanto, el uso de redes sociales en contextos educativos no tiene que limitarse únicamente a la educación secundaria o superior. Autores como Gómez y Redondo (2011) relacionan la importancia de iniciar la aproximación a las redes sociales en estas edades tan tempranas, con "la necesidad de enseñar al alumnado las consecuencias que pueden tener sus actuaciones con las redes sociales, por lo que se les debe de ofrecer unas pautas de comportamiento" (p.10) con la intención de que se haga un buen uso de ellas, ya que no todo son ventajas al poder presentarse determinados riesgos asociados a su utilización, debido a un uso descontrolado e inadecuado por los más jóvenes, por lo que surgen propuestas siempre orientadas a la "alfabetización mediática que ayuden a racionalizar el uso de las redes y adoptar una postura crítica frente a ellas, por entender que desde la educación se pueden aprovechar las oportunidades que ofrecen y prevenir los riesgos asociados" (Del Moral y Villalustre, 2012, p. 42).

\section{METODOLOGÍA}

Multitud de estudios llevados a cabo por diferentes autores como Abarca (2013), Fernández (2012), Gutiérrez, Sánchez y Castañeda (2013), Moratón, Luna y Romero (2015), Muñoz. Fragueiro y Ayuso (2013), Pérez, Ortiz y Flores (2015), avalan las posibilidades y las ventajas de la incorporación de las redes sociales en la educación, por lo que podríamos plantearnos 
por qué estas no se implementan de manera cotidiana en el aula y más concretamente en la de primaria.

Esta investigación, que tiene un carácter exploratorio y descriptivo, trata de dar respuesta al propósito principal de la misma, conocer cuál es la percepción que tienen los docentes sobre el uso de las redes sociales en el aula de primaria en un centro educativo de la Región de Murcia y los objetivos de la misma son:

1.Explorar y describir la percepción de los docentes sobre el uso de las redes sociales en el aula de primaria, en un centro educativo de la Región de Murcia.

2. Acercar a los docentes las potencialidades que las redes sociales pueden aportar en el ámbito educativo, a través de la implementación de una propuesta de innovación docente basada en el uso de una red social educativa.

3. Analizar tras el desarrollo de la experiencia, si se ha modificado la percepción de los docentes sobre el uso de las redes sociales en el aula de primaria.

Este estudio se integra dentro de dos modelos de investigación, por un lado se corresponde con un estudio de caso, puesto que se ha examinado un conjunto de acontecimientos, actividades e individuos en un tiempo y en un lugar determinado y por otro lado, también se encuentra integrada en la denominada Investigación Basada en Diseño (IBD), ya que es "una investigación con implicaciones sobre la práctica, cuyo énfasis es la solución de problemas y la construcción de conocimientos dirigido al diseño, desarrollo y evaluación del proceso educativo" (de Benito y Salinas, 2016, p.46), ocupándose de problemas reales que han sido identificados en la práctica docente del centro educativo; y que ha pretendido mejorar la calidad instructiva en los diferentes niveles y contextos educativos. Por ello, se ha diseñado una experiencia de innovación educativa basada en la creación y uso de una red social educativa; impartiendo además un seminario de formación docente para hacer una primera aproximación a las posibilidades educativas de las TIC y de las redes sociales en los entornos educativos. Además, se lleva a cabo la creación de una red social en el centro en la que se realizan una serie de actividades para que los docentes conozcan de primera mano las posibilidades que ofrecen estas herramientas. La idea principal es que esta red quede creada en el centro y que sean los propios docentes, los que después de la experiencia continúen gestionando y generando contenidos, recursos y proyectos que les ayuden a una implementación eficaz de las RRSS educativas.

La red utilizada en esta experiencia ha sido Edmodo. Alonso, Morte y Almansa (2015) la destacan por encima de las demás, al presentar dos ventajas fundamentales sobre el resto: por un lado, que los alumnos solo precisan dar su nombre para acceder a la misma, al crear el profesor grupos privados y generar códigos de acceso y por otro lado el control parental que permite a las familias "acceder al calendario, notas, mensajes del alumno y enviar o recibir mensajes del profesor" (p.98).

El escenario de intervención elegido para llevar a cabo esta investigación es un colegio concertado de la Vega Alta del Segura, y se lleva a cabo en la etapa de primaria. El centro dispone de una sola línea, por lo que se interviene con los profesores integrantes del equipo docente de dicha etapa educativa. Debido a esta estructura organizativa del centro, la muestra con la que trabajaremos será de un total de $\mathrm{N}=12$ docentes. 
El estudio se desarrolló a lo largo de cuatro fases entre las que se incluyen una fundamentación teórica; un análisis previo del centro educativo, de los recursos y de la formación del profesorado, al que se le facilitó un cuestionario inicial para detectar el nivel perceptivo del que partimos. Posteriormente se impartió un seminario formativo sobre las posibilidades educativas de las RRSS y se creó la red social señalada anteriormente, para que pudieran comprobar cómo se gestiona; finalizando la experiencia con la realización de un cuestionario final para comparar y poder detectar si todo el proceso ha conseguido mejorar la percepción sobre el uso de las RRSS de que partíamos inicialmente.

Los datos, por tanto, se han obtenido a través de dos cuestionarios (inicial y final), elaborados y validados dentro del Grupo de Investigación en Educación y Nuevas Tecnologías de la Universidad a Distancia de Madrid (UDIMA), una ficha de registro de observaciones (observación participante) y con datos recogidos de Edmodo en un diario del investigador. En cuanto al modelo de recogida de datos, estamos hablando de un enfoque mixto, ya que como señalan Johnson y Onwuegbuzie, (2004, p.17) "los modelos mixtos de investigación son formalmente definidos como una clase de investigación donde el investigador mezcla o combina técnicas, métodos, aproximaciones, conceptos y lenguaje cuantitativos y cualitativos en un estudio simple".

\section{RESULTADOS}

\subsection{Resultados del cuestionario inicial}

Del total del profesorado participante, el $50 \%$ señala que hace uso de las RRSS varias veces a la semana. En cuanto a las redes sociales educativas, podemos señalar que las más conocidas y usadas son Internet en el Aula (3), Edmodo (1), RedAlumnos (1), Edu 2.0 (1) y Skype in the Classroon (1) (Figura1). El uso que se hace de las RRSS es eminentemente a título personal, siendo su uso prioritario las actualizaciones de estado y visitar perfiles de contactos, como así lo señala el $100 \%$ de los participantes. Es muy significativo que de entre las diferentes opciones planteadas, salvo la organización de eventos, solo uno de los docentes las haya considerado como muy útiles, y que tres de ellos hayan considerado como nada útil la opción de divulgar resultados.

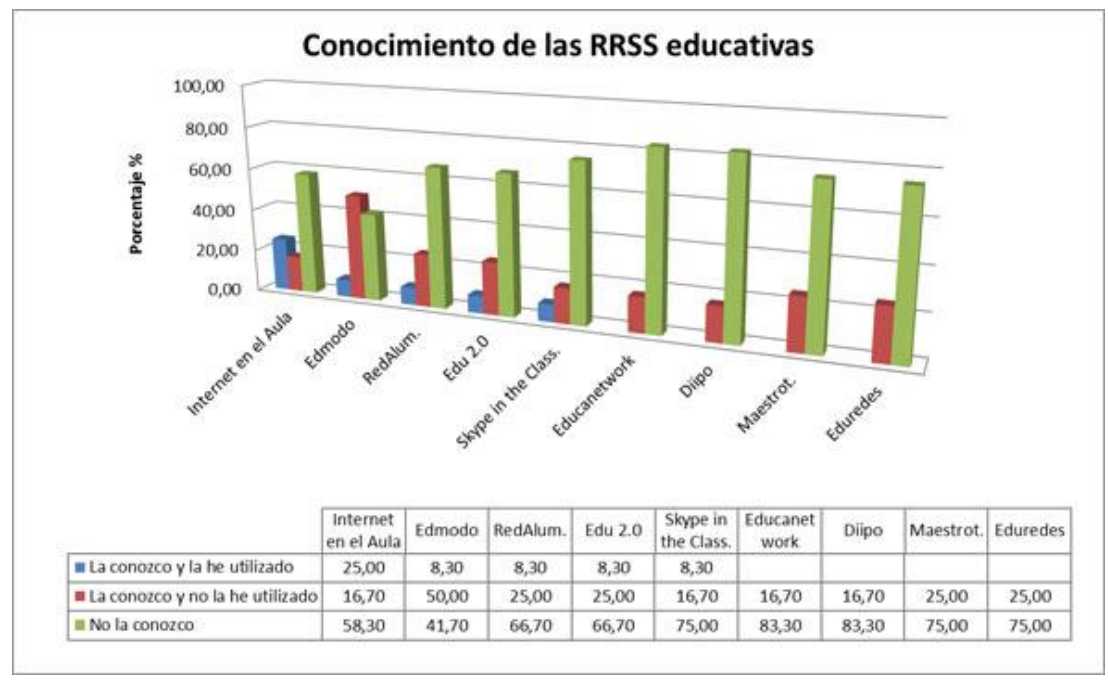

Figura 1. Conocimiento y uso de las RRSS educativas. 
Planteadas las mismas opciones en la interacción de los docentes con los padres de los alumnos, encontramos que señalan como muy útil la posibilidad de hacer un seguimiento del alumno (8), acordar reuniones (7), enviar y recibir mensajes (4) y compartir materiales adicionales (2). En cuanto a la capacidad y formación que los docentes se atribuyen a sí mismos para implementar el uso de las RRSS en el aula, encontramos que unos pocos se ubican en puntuaciones altas ( 3 casos repartidos entre el 8 , el 9 y el 10), pero que la mayoría se ubican en valoraciones del 5 hacia abajo, encontrando la mayoría ( 5 casos) entre el 3 y el 4.

Cuando se les consulta acerca de qué herramientas de las que proporcionan las RRSS conocen los docentes, han señalado como más significativas la personalización del perfil personal (9), el muro de actualizaciones (8), y los foros de opinión (7). También se ha tratado de conocer qué características son las que suponen rechazo o miedo hacia el uso de las RRSS educativas, señalando como más características la difusión de imágenes y vídeos (12) y el poco control en las conversaciones de los alumnos (11), seguidas de la necesidad de crear email para su uso y la falta de control parental con ocho docentes para cada una de las opciones (Figura 2).

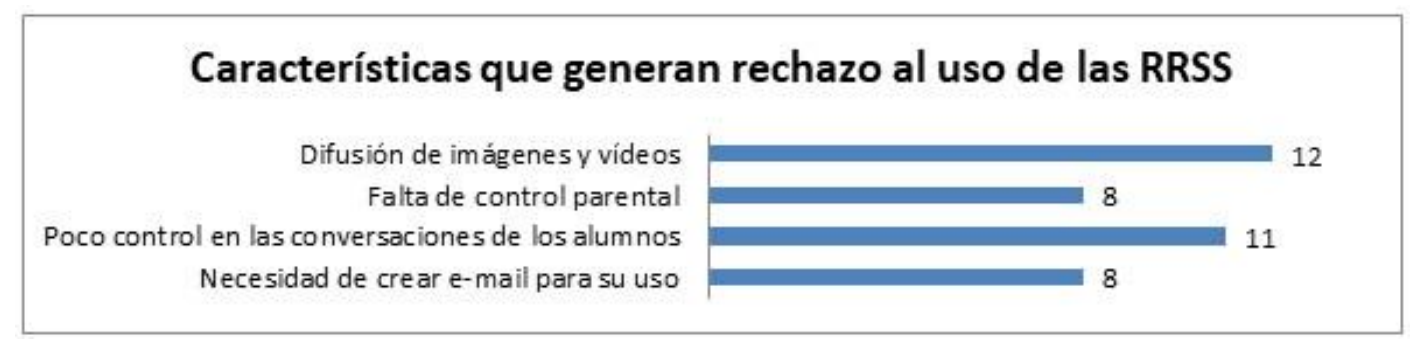

Figura 2. Rechazo del profesorado al uso de la RRSS.

También se ha procurado conocer de qué dispositivos tecnológicos disponen los docentes, tanto a nivel personal como educativo, en donde encontramos que el ordenador es usado mayoritariamente en ambos sentidos (8), y la Tablet es usada mayoritariamente a título personal (7), siendo solamente dos los docentes que la utilizan en ambos aspectos y que el Iphone/Smarthone se usa exclusivamente a título personal, siendo nulo su uso a título educativo.

En cuanto a la valoración sobre qué dificultades más evidentes evitan el uso de las RRSS en el aula, encontramos que la falta de recursos (7) y la falta de formación (9) son las opciones más señaladas.

En cuanto a las principales ventajas y desventajas que detectan en el uso de las RRSS en la práctica docente, se han obtenido como resultados:

- Cuatro docentes señalan como ventaja principal una comunicación ágil y eficaz entre los usuarios.

- Dos señalan ventajas asociadas con el uso de contenidos.

- Y por último, tres señalan que desconocen las ventajas que plantean este tipo de herramientas.

En cuanto a las desventajas más significativas se centran en dos aspectos fundamentalmente: 
- Un uso descontrolado por parte de los alumnos (3).

- Falta de privacidad para todos los participantes en la red (3).

\subsection{Resultados del diario del investigador}

Tras la implementación de la formación y la propuesta de innovación trabajada con ellos, se ha podido comprobar como los docentes de las tres asignaturas incluidas inicialmente para llevar a cabo esta propuesta (Ciencias Naturales, Matemáticas y Lengua Castellana), han ido incluyendo contenidos no solo para las unidades didácticas previstas inicialmente, sino para las que han ido realizando hasta la finalización del curso escolar.

La presentación de las tareas en el muro de la plataforma por parte del alumnado, tanto las individuales como las grupales, ha sido fluida y ha cumplido con las expectativas que se habían planteado inicialmente puesto que no ha habido problemas destacables a la hora de su presentación. En este sentido los docentes han señalado, como se desprende de los datos recogidos mediante la observación participante, que inicialmente no tenían mucha confianza sobre una participación eficaz del alumnado, y, aunque los alumnos han requerido algunas ayudas, la experiencia ha sido positiva puesto que han podido comprobar desde el inicio de las unidades cuales eran los mayores problemas conceptuales que se presentaban en el alumnado y la capacidad de este para enfrentarse a estas herramientas, a pesar de que en algunas ocasiones percibían que se perdía el control del aula. También hacen una referencia positiva en cuanto a las posibilidades comunicativas y evaluativas que presenta la plataforma en torno a las diferentes actividades que se pueden plantear y que sería interesante iniciar proyectos colaborativos con otros centros.

Por otro lado, el resto de docentes de primaria han estado experimentando con sus asignaturas en la plataforma, incluyendo contenidos y recursos y haciendo un seguimiento del proceso llevado a cabo en las asignaturas planteadas. Además, después de la experiencia llevada a cabo para este estudio, han utilizado estos contenidos en las últimas unidades de sus asignaturas a través de la red creada, señalando además que la implementación de estos contenidos les será válido para el próximo curso escolar.

\subsection{Resultados del cuestionario final}

El objetivo principal de este cuestionario era tratar de averiguar si una vez utilizada la red social en el aula estarían dispuestos a incluir una red social educativa en su metodología diaria, obteniendo como resultado que 8 de los 12 participantes, sí estarían dispuestos a su inserción en el aula, por el contrario 4 continuarían sin implementar ninguna de estas redes en su aula. Cuando se les consulta sobre los motivos de su respuesta, indican principalmente que han podido comprobar las ventajas que pueden llevar asociadas tanto metodológicamente como en cuestión de recursos (5 casos), aunque 3 de ellos señalan que siguen siendo reacios a la incorporación de las RRSS en el aula por diferentes motivos.

Con otro de los ítems de este cuestionario, se trata de conocer en qué medida les parecen útiles las RRSS, después de la experiencia llevada a cabo para el trabajo docente, encontrando que la mayoría se sitúa en los índices superiores de valoración (Figura 3). 


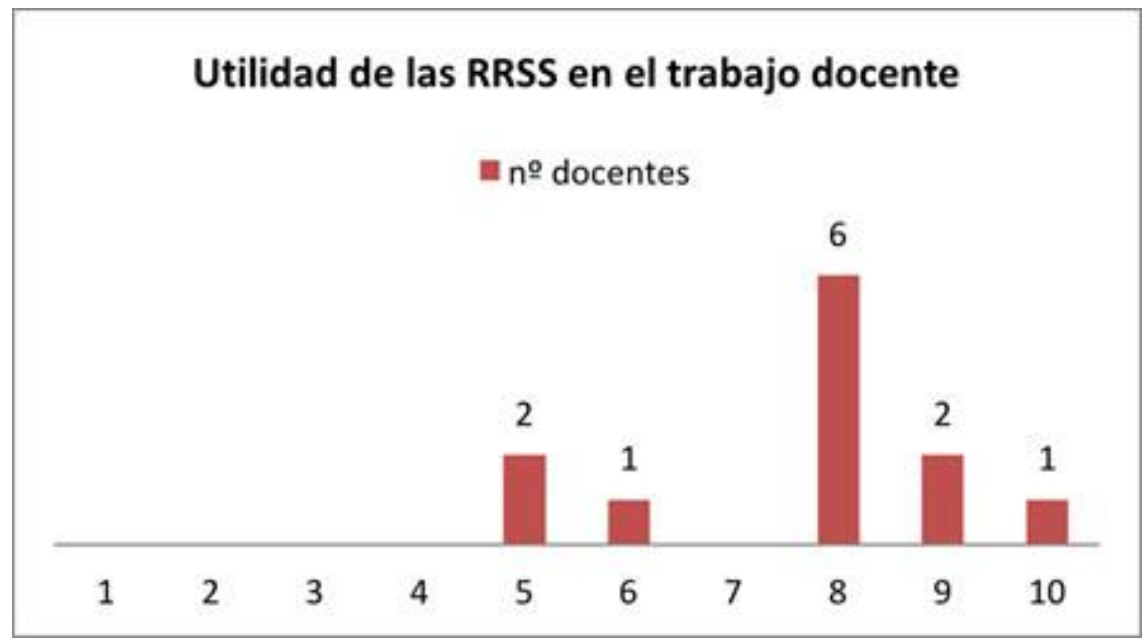

Figura 3. Utilidad educativa atribuida a las RRSS después de la experiencia.

Cuando se les preguntaba acerca de su capacidad para utilizar las RRSS, su percepción se ha situado por encima de la mitad de la misma siendo el seis y el siete los niveles más señalados con tres individuos en cada uno de ellos y situándose los niveles dos, tres y cuatro con una persona en cada uno de ellos como los más bajos.

Cuando se les consulta sobre qué han descubierto tras la experiencia, la respuesta más significativa es la posibilidad de inclusión de recursos y la privacidad de la que disponen las redes sociales educativas, ya que ha sido señalada por nueve de los docentes. Aunque cabe destacar que tres de estos docentes han señalado que la introducción de este tipo de herramientas requiere una formación continua y un mayor trabajo docente fuera del horario escolar.

En cuanto a las ventajas que atribuyen los docentes a las RRSS educativas después de haber trabajado con ellas, el total de participantes, presenta como más significativa la posibilidad de la integración de otras herramientas TIC como son los blog, vídeos, imágenes, cuadernos de notas, etc. en una sola plataforma, 10 de los docentes señalan como ventaja el entorno sencillo, intuitivo y común para profesores y alumnos y siete de ellos señalan la transparencia que ofrecen respecto a los padres/tutores.

En cuanto a cómo incluirían los docentes las RRSS educativas en el centro educativo, encontramos que un total de 10 docentes señalan que realizarían esta incorporación a través de un proyecto integral que emane desde el centro y que sea gestionado por un profesional, los otros dos señalan que a través de grupos individualizados y privados como el que hemos realizado en la experiencia llevada a cabo en el centro.

En cuanto a los aspectos formativos relacionados con RRSS, se pretendía conocer si el proceso formativo que se ha llevado a cabo a lo largo de esta investigación ha generado en los docentes la inquietud de formarse en el uso de estas herramientas. Encontramos que 11 de ellos señalan que sí y solo uno todavía es contrario a recibir dicha formación. Para averiguar el porqué de estas respuestas, este ítem se completa con una pregunta abierta en la se debe de contestar las razones que condicionan la respuesta inicial. Las respuestas se engloban en tres opciones principalmente, siendo la más señalada la referida a la 
potencialidad observada a lo largo de las experiencias llevadas a cabo en el centro educativo (8 docentes), seguida de la posibilidad de ampliación de conocimientos en las TIC (3) y un último individuo que señala que no se formaría porque aún no está interesado en la implementación de estas herramientas.

Finalmente, se pretende conocer qué es lo más les interesaría aprender sobre las RRSS educativas para poder implementarlas en su práctica cotidiana. Revisadas y agrupadas todas las opciones que han señalado, tenemos que "conocer todas sus utilidades" (4) y las "posibilidades de interacción con los alumnos, padres y equipo docente" (4) se han convertido en las más referenciadas.

\section{CONCLUSIONES Y DISCUSIÓN}

Se ha observado que los docentes, objeto de estudio, presentan una percepción muy baja sobre las posibilidades de la incorporación de las redes sociales en el aula, ya que el uso que hacían antes de la experiencia de innovación era escaso y cuando lo hacían, giraba en torno a aspectos relacionados con su perfil personal y de sus propios contactos, y todo ello a través de redes sociales de las denominadas generalistas $u$ horizontales. También hemos encontrado que desconocen por norma general las RRSS educativas y los recursos que pueden proporcionar; todo motivado por la falta de formación y en algunos casos por la falta de implicación y motivación que presentan al señalar que les supone un esfuerzo añadido a su trabajo docente. Lo que les lleva a desconocer las ventajas y posibilidades que presentan las RRSS en entornos educativos. Resulta significativo que acciones como la promoción de actividades y logros conseguidos, quede en los últimos puestos asignados a los usos de estas herramientas.

Con la realización de experiencia educativa llevada a cabo en el centro, se ha producido un cambio tanto en la predisposición de uso como en la utilidad que atribuían los docentes a las RRSS, lo que ha supuesto un cambio significativo en la percepción inicial de los docentes sobre las RRSS. Los participantes han valorado positivamente la información, las sugerencias y las recomendaciones recibidas. Aunque al final de la experiencia siguen demandando más formación y apoyo de profesionales que les ayuden a obtener una capacitación eficaz en el uso de las TIC. Ha quedado, por tanto, en evidencia que la formación continua es crucial en todo proceso de integración de nuevos elementos en los procesos formativos y que en la mayoría de ocasiones esta falta de formación genera prejuicios preconcebidos que impiden poner en marcha nuevos procesos que nos hagan evolucionar profesionalmente, algo muy importante en un mundo educativo que no para de cambiar en base a nuevos conceptos estructurales, formativos y pedagógicos.

También hemos encontrado en algunos casos un rechazo, aunque de forma aislada, hacia la experiencia en general y el uso de la red social en particular. Un rechazo a lo desconocido, a lo cambiante y a algo que necesita una dedicación, esfuerzo y formación continua. En este sentido García (2008) se lamenta de que este rechazo se traduzca en una escasa investigación sobre el rendimiento educativo y que además este mismo sentimiento se transfiera a los docentes y desconfíen de la tecnología utilizada por los estudiantes, ya que de un modo $u$ otro interfiere en el proceso de enseñanza formal. 
Por lo que las propuestas de mejora, de esta situación, deben pasar necesariamente por el compromiso explícito de los docentes y una apuesta por el uso de herramientas que nos ayuden a estos cambios metodológicos, sin perder de vista la perspectiva de que las RRSS no deben verse como un remedio, sino como una herramienta que controlen los contenidos y la comunicación síncrona y asíncrona entre docentes y alumnado en el proceso educativo (Abarca, 2013).

Debemos ser conscientes que estos cambios mejorarán también nuestras propias capacidades dentro del proceso de enseñanza-aprendizaje. Por eso y partiendo de esta premisa, se debe de replantear la formación inicial del profesorado, así como su formación continua, tratando de adaptarla a una realidad social, cultural y educativa en cambio permanente. Lo que requiere un esfuerzo considerable por su parte para asumir estas necesidades formativas y de alfabetización digital con la intención de generar en ellos mismos las competencias necesarias que faciliten esta tarea. Para ello, deben de reflexionar sobre su entorno personal de aprendizaje (PLE), donde intervienen herramientas, servicios que permiten el acceso y la relación con la información y la interacción con otras personas; en definitiva: ¿dónde aprenden?, ¿cómo aprenden? y ¿con quién aprenden? (Adell y Castañeda, 2010).

\section{REFERENCIAS}

Abarca, S. (2013). Las redes sociales como instrumento de mediación pedagógica: alcances y limitaciones. Actualidades Investigación en Educación, 13(2). Recuperado de http://www.redalyc.org/pdf/447/44727049013.pdf

Adell, J. y Castañeda, L. (2010). Los entornos personales de aprendizaje (PLEs): una nueva manera de entender el aprendizaje. En R. Roig y M. Fiorucci (Eds.), Claves para la investigación en innovación y calidad educativas. La integración de las Tecnologías de la Información y la Comunicación y la Interculturalidad en las aulas. Alcoy: MarrilRoma URE Univertita degli studi. Recuperado de https://digitum.um.es/xmlui/handle/10201/17247

Alemany, C. (2009). Redes sociales: una nueva vía para el aprendizaje. Cuadernos de Educación y Desarrollo, 1(1). Recuperado de http://www.eumed.net/rev/ced/01/cam4.htm

Alonso, S., Morte, E. y Almansa, S. (2015). Redes sociales aplicadas a la educación: Edmodo. Revista de Educación Mediática y TIC (edmetic) 4(2), 88-11. Recuperado de https://dialnet.unirioja.es/descarga/articulo/5192042.pdf

Camacho, M. (2010). Las redes sociales para enseñar y aprender. Reflexiones pedagógicas básicas. En L. Castañeda (Coord.), Aprendizaje con redes sociales. Tejidos educativos para los nuevos entornos (pp. 91-104). Sevilla: MAD.

De Benito, B. y Salinas, J. (2016). La Investigación Basada en Diseño en Tecnología Educativa. Revista Interuniversitaria de Investigación en Tecnología Educativa RIITE, 0. Recuperado de http://revistas.um.es/riite/article/view/260631/195691 
Del Moral, M.E. y Villalustre, L. (2012). Presencia de los futuros maestros en las redes sociales y perspectivas de uso educativo. Revista Latinoamericana de Tecnología Educativa (RELATEC). 11(1), 41-51. Recuperado de http://relatec.unex.es/article/viewFile/843/633

Espuny, C., González, J., Lleixá, M., Gisbert, M. (2011). Actitudes y expectativas del uso educativo de las redes sociales en los alumnos universitarios. En: El impacto de las redes sociales en la enseñanza y el aprendizaje [monográfico en línea]. Revista de Universidad y Sociedad del Conocimiento (RUSC). 8 (1), 171-185. Recuperado de http://www.raco.cat/index.php/Rusc/article/viewFile/225630/306986

Fernández, T. (2012). Aprendizaje colaborativo y uso de las redes sociales en Educación Primaria. Recuperado de http://revistas.ucm.es/index.php/DIDA/article/viewFile/42240/40214

García, A. (2008). Las redes sociales como herramienta para el aprendizaje colaborativo: una experiencia con Facebook. Revista RE-Presentaciones, 5, 49-59. Recuperado de https://dialnet.unirioja.es/servlet/articulo?codigo=3129947

Gómez, J.A. y Redondo, C. (2011). Las redes sociales como fuente de conocimiento en la enseñanza primaria. XII Congreso Internacional de Teoría de la Educación (CITE) 2011, Barcelona. Texto completo recuperado de http://www.cite2011.com/Comunicaciones/TIC/150.pdf

Gutiérrez, I., Prendes, M.P. y Castañeda, L. (2015). Aprendizajes y competencia digital. En J. Cabero y J. Barroso (Coords.), Nuevos retos en tecnología educativa (pp. 239-256). Madrid: Síntesis.

Gutiérrez, I., Sánchez, M.M. y Castañeda, L. (2013). Nuevos medios, procesos y materiales de trabajo en las aulas de educación infantil y primaria. En M. C. Martínez Serrano. (2013), Buenas prácticas educativas en el uso de las TIC (pp. 75-106). Jaén: Joxman. Recuperado de https://digitum.um.es/jspui/bitstream/10201/41847/1/Gutierrez_Sanchez_Castaneda _2013.pdf

Johnson, R. \& Onwuegbuzie, A. (2004). Mixed Methods Research: A Research Paradigm Whose Time Has Come, 33(7), 14-26. Recuperado de http://www.socsci.uci.edu/ castellj/sshonors/webdocs/research_design/Mixed\%20M ethods\%20Research.pdf

Marquès, P. (2008). Las competencias digitales de los docentes. Recuperado de http://peremarques. pangea.org/competenciasdigitales.htm

Moratón, B., Luna, M. y Romero, J. (2015). Las redes sociales en la Educación Primaria: conocimiento y uso por parte de maestros participantes en un entorno formativo. Tecnología, ciencia y educación. 1, 77-83. Recuperado de http://www.tecnologiaciencia-educacion.com/index.php/TCE/article/view/31 
Muñoz, M.M., Fragueiro, M.S. y Ayuso, M.J. (2013). La importancia de las redes sociales en el ámbito educativo. Recuperado de https://dialnet.unirioja.es/descarga/articulo/4425349.pdf

Pérez, M.S., Ortiz, M.G. y Flores, M.M. (2015). Redes sociales en Educación y propuestas metodológicas para su estudio. Ciencia, docencia y tecnología, 26(50), 188-206. Recuperado

http://rua.ua.es/dspace/bitstream/10045/11902/1/articulo\%202\%202009.pdf

Prendes, M.P. y Gutiérrez, I. (2011). Competencias tecnológicas del profesorado en las universidades españolas. Revista de educación, 361, 196-222. doi: 0.4438/1988-592XRE-2011-361-140

Sloep, P. \& Berlanga, A. (2011). Learning Network, Networked Learning. Comunicar, 37(19), 55-64.

Recuperado

de https://www.revistacomunicar.com/index.php?contenido=detalles\&numero=37\&arti culo=37-2011-07

\section{Para referenciar este artículo:}

Ibáñez Zapata, P., \& Sánchez Vera, M. (2019). Percepción de los docentes sobre el uso de las redes sociales en el aula de Primaria. Una experiencia en un centro educativo de la Región de Murcia. Edutec. Revista Electrónica De Tecnología Educativa, (66). https://doi.org/10.21556/edutec.2018.66.1219 